\title{
Debriefing of the medical team after emergencies on cruise ships
}

\author{
Eilif Dahl \\ Department of Occupational Medicine, Norwegian Centre for Maritime and Diving Medicine, \\ Haukeland University Hospital, Bergen, Norway
}

\begin{abstract}
Done to improve safety and patient outcome but not to lay blame, debriefings on cruise ships should preferably be conducted as standard practice in the medical facility immediately after all critical events aboard. The key questions to be asked are: What went well, what could have gone better and what must participants do to improve care? Post-debriefing the ship's doctor might have to deal with team members' mental stress resulting both from the event and from debriefing it.

Required by most cruise companies, standardised advanced life support courses teach effective high -performance team dynamics. They provide the multinational medical staff with a clearer understanding of the rescue sequence, which again will reduce the risk of mistakes and simplify post-event debriefings. Their systematic approach to the chain of survival is also helpful for post-event debriefings if something went wrong.
\end{abstract}

(Int Marit Health 2017; 68, 4: 183-186)

Key words: debriefing, cruise ships, emergency, medical teamwork, life support courses, communication, maritime medicine

Feedback is the breakfast of champions

(Ken Blanchard)

\section{A SITUATION}

"Code Alpha" (= medical emergency) is called over the public announcement (PA) system on a cruise ship. The medical team members rush to a cabin where a passenger has just seen her 72-year-old husband lose consciousness. When the responders arrive, he is in cardiac arrest. He is successfully resuscitated and is then transported to the onboard medical facility for further stabilisation. Not all actions were uneventful and a responder asks for a debriefing. What does debriefing after a medical event on a cruise ship entail and why, where, when, how and by whom should it be done?

\section{BACKGROUND}

Debriefing is defined as a meeting where someone gives a report about an important job that they have just finished [1]. On ships the Master (Captain) routinely conducts debriefings immediately after drills and critical events in order to improve response operations [2].
All cruise ships must have a doctor [3] and depending on vessel size and itinerary they will have a medical team that may comprise not only the ship's doctor(s), but also 2-5 other medical professionals (nurses, paramedics) and a non-medical stretcher team. The stretcher team consists of crewmembers more or less randomly assigned to stretcher duty by the safety officer and trained by the medical staff in basic first aid and techniques for transport of injured and ill patients aboard.

All medical team members are mandatory participants in all ship drills that might involve medical conditions (fire, man-over-board, bomb search etc.). During the drills the Master is provided with frequent updates from the various teams by phone and walkie-talkie and the drills work as training for the medical team members in interdepartmental communication and cooperation and in patient transport through demanding areas of the vessel. After each drill the senior officers participate in the Master's debriefing on the 
Bridge. There the (senior) doctor will provide feedback if major challenges were encountered or external assistance was needed. Most of these debriefings are concluded with a short statement like "the medical part of the drill was uneventful".

According to ship programs, medical emergency drills are to be held every 2-4 weeks, but although they have prepared the medical team members for various scenarios, much can go wrong in real emergencies. The critical events are usually announced by code over the PA system from the Bridge. All medical team members will rush to the event location and there stabilise the patient well enough for fast but safe transport to the medical facility aboard for further diagnostic work-up and treatment. Unexpected challenges occurring along the way must be handled as well as possible. Afterward they must be addressed in a thorough but blameless debriefing of all involved to ensure that mistakes are understood and corrected - and will not recur during the next emergency.

Based on a practical guide for initiation of debriefing programmes in emergency departments ashore by Kessler, Cheng and Mullan [4], this article may help medical teams to start debriefing after medical emergencies and drills involving patient management on cruise ships.

\section{FACTORS TO PREVENT ERRORS}

Keys to uneventful and successful handling of emergency situations aboard are good pre-event preparation: reliable reporting systems [5], comprehensive ready-to-go mobile emergency equipment [6], a well-organised medical centre [7, 8], a coordinated high-performance medical team [9], and systematic debriefing after every critical event that involves medical staff.

All cruise ship doctors and nurses must have postgraduate emergency medicine training $[7,8]$. But they come from all over the world and have different cultural backgrounds and language skills. Therefore the universal recommendation of Basic Life Support (BLS), Advanced Cardiovascular Life Support (ACLS), Paediatric Advanced Life Support (PALS) and Advanced Trauma Life Support (ATLS) certification of all medical staff on cruise ships every 2 years is essential for effective teamwork $[7,8]$. These standardised courses emphasize a strict systematic approach to assess and treat respiratory or cardiac dysfunction, followed by searching for and treating underlying causes [9]. During practical exercises of various critical cardiac scenarios by using standardised algorithms, it is repeatedly demonstrated how essential teamwork is for a positive outcome. All course participants must show that they can perform satisfactorily and in a timely fashion the various team roles of megacodes. Every participant must also be the megacode team leader and demonstrate understanding of effective high-performance team dynamics by assigning clear roles and responsibilities while taking into consideration the team members' limitations and capabilities. All the team members are encouraged to give clear messages and share their knowledge by "constructive intervention" and "closedloop communication" while showing mutual respect for each other [9].

The standardised life support courses provide multinational team members with a clearer understanding of the rescue sequence, which again can reduce the risk of mistakes and simplify necessary post-event debriefings.

\section{WHY AND WHAT?}

Debriefing on ships is done to detect and discuss problems that occurred when dealing with a critical situation to improve future performance. An important part is to praise things well done - and to deflate guilt or blame. Thus the atmosphere must be friendly and supportive without any finger pointing or search for scapegoats. Reflections of the team on knowledge, attitudes, skills or teamwork behaviours demonstrated during the event may help to recognize and understand contributions to an error as well as how to replicate successful team performance $[4,10]$.

In emergency departments ashore the trigger for debriefing may be a complex decision process, whereas at least on smaller ships - where medical events are relatively rare - the decision should be simple: all medical drills, all code calls, all emergency encounters necessitating medical support outside the ship's medical facility, and all ward admissions warrant a debriefing. Debriefings should be viewed as golden opportunities to review and update procedures that are infrequently practiced on board. "Unnecessary" code calls or alarms also merit debriefings, primarily to find ways to reduce potential overutilization of the code system [5].

On land psychological debriefing has become a widespread and expected intervention after trauma exposure to decrease the likelihood of psychological dysfunction, from mild depression to posttraumatic stress syndrome [11]. Professional psychological debriefing at sea is unrealistic, but sensitive attempts to remove feelings of failure or guilt and to restore confidence in the participants are worthwhile. Defusing, whose sole purpose is venting emotions to reduce tension [4], may sometimes be helpful.

Furthermore, defusing or quickly debriefing after non-urgent events when there have been arguments or a team member has felt abused or ignored may quickly clear the air. The small medical teams must continue to live and work together for months, and if bad feelings between them are left to fester, the rest of their contracts will emotionally be ruined and the resulting lack of communication may easily become a safety hazard. 
After a debriefing, team members may feel distressed both because of the event and the debriefing itself. Medical sign-off or compassionate leave are options to consider if this cannot be resolved satisfactorily aboard by the medical team, the department head, the human resources manager and/or friends and colleagues.

\section{WHO?}

\section{DEBRIEFING PARTICIPANTS}

Not only the medical team members but all ship employees who were involved in the event and can contribute to or learn from the debriefing should be invited. The debriefing should start with discussing mishaps outside the medical facilities, like alarm procedures and crowd control, so that the non-medical participants who are expected back to work can be dismissed early.

\section{DEBRIEFING FACILITATOR}

The (senior) doctor will be expected to conduct medical debriefings, but an authority figure may inhibit or bias the discussion. A nurse who gets along well with all the team members may occasionally be a better choice. In particular if there have been vocal disagreements or misunderstandings during the event, it may be necessary to bring in outside facilitators, like the staff captain or the ship's human resources manager, alternatively arrange a teleconference with the company's medical consultant ashore.

\section{WHEN AND WHERE?}

Kessler, Cheng and Mullan use temperature adjectives to classify the timing of debriefing: "hot" (immediately), "warm" (minutes to hours after the event) and "cold" (days to weeks later) debriefings [4].

On cruise ships hot, short and to-the-point debriefings are preferable when feasible. The favoured location is the temporarily closed medical facility, as this is where the entire team will routinely gather after all clinical events. Then recall bias is minimized, and urgent issues identified during the debriefing can immediately be addressed.

Although in other settings a commonly perceived barrier is insufficient time, most debriefings take less than 10 $\min [10,12]$. Experienced ship's doctors have stated that hot debriefing may only be an option "if the patient died or recovered from a faint" and after drills; in all other circumstances most or all of the team members are too occupied treating the patient to be able to debrief.

Later reassembling of all involved, cold debriefings, will even on a small vessel be a logistical challenge. They are usually only attempted if team members are reluctant to share information about suboptimal care before they can get legal advice or if the company fears medico-legal reactions.
Issues that were revealed but could not be corrected during the event or the debriefing must be followed up until resolved.

\section{HOW?}

Many methods have been described for doing debriefings ashore [4]. On board debriefings should be systematic but simple: Go through the event step by step (emergency call, arrival at the scene, findings and stabilization at the scene, patient transport to the medical facility, findings and further treatment there, transfer to medical facility ashore) and ask at each step: What went well, what could have gone better and what must participants do to improve care?

The focus here is not simply on patient outcomes (e.g. patient survived or died) but on the structures (e.g. broken stretcher) and processes (e.g. AED didn't charge) that contributed to the outcomes [13].

Some pitfalls to avoid may include tangential discussions (e.g. generating lists of mistakes without dissecting the underlying rationale), turning the debriefing into a blame session, and leaving team members out of the discussion [12].

When national transport safety investigators conduct debriefings, the information given by participants is classified as 'restricted information', which is exempt from disclosure. To encourage an open flow of information in the interest of future transport safety, the law prevents it from being admitted as evidence against the person in any civil or criminal proceeding [14]. It should be noted that debriefing information on cruise ships is not similarly protected. Although ethical standards are emphasized by cruise companies, there is sufficient anecdotal evidence from ships to make crewmembers suspect disparity between official policy and ethical practice. The recent trend to employ medical staff on cruise ship rather than keep them as independent contractors will at least get them better legal protection and should also reduce their fear of individual malpractice law suits [15].

\section{CONCLUSIONS}

Done to improve safety, future performance and patient outcome but not to lay blame, debriefings on cruise ships should preferably be conducted in the medical facility as standard practice immediately after all medical drills and emergencies aboard. The key questions to be asked are: What went well, what could have gone better and what must participants do to improve care?

Issues that were revealed but could not be corrected during the event or the debriefing must be followed up until resolved. Post-debriefing the ship's doctor might have to deal with team members' mental stress resulting both from the event and from debriefing it. 
Standardised advanced life support courses teach effective high-performance team dynamics to ensure uniform state-of-the art treatment of cardiovascular emergencies. Required by most cruise companies, they provide the multinational medical staff with a clearer understanding of the rescue sequence, which again will reduce the risk of mistakes and simplify post-event debriefings. Their systematic approach to the chain of survival is also helpful for post-event debriefings if something went wrong.

\section{POTENTIAL CONFLICT OF INTEREST}

The author has no commercial, financial or other relationships related to the subject of this article. He has worked as ship's doctor and medical consultant for many cruise companies.

\section{REFERENCES}

(Internet references accessed 28 August 2017)

1. Macmillan Dictionary. Debriefing. http://www.macmillandictionary. com/dictionary/american/debriefing.

2. International Maritime Organisation (IMO). Sub-committee on human training and watch keeping, 3rd session, (HTW/3). Review of STCW passenger ship-specific safety training. Specification of minimum standard of competence in crisis management and human behaviour. Table A-V/2-2; 2015. .https://www.cruising. org/docs/default-source/imo/proposed-amendments-to-the-stcw-convention-passenger-ship-specific-safety-training.pdf?sfvrsn $=0$.

3. International Labour Organization. Maritime Labour Convention, 2006. .http://www.ilo.org/wcmsp5/groups/public/@ed_norm/ @normes/documents/normativeinstrument/wcms_090250.pdf.

4. Kessler DO, Cheng A, Mullan PC. Debriefing in the emergency department after clinical events: a practical guide. Ann Emerg Med. 2015;
65(6): 690-698, doi: 10.1016/j.annemergmed.2014.10.019, indexed in Pubmed: 25455910.

5. Taylor CJ. Medical emergency announcements on cruise ships: an audit of outcome. Int Marit Health. 2015; 66(4): 203-206, doi:10.5603/IMH.2015.0040, indexed in Pubmed: 26726890.

6. Dahl E, Diskin A, Giusti AC, et al. A first response bag with standardized contents for medical emergencies on cruise ships. Int Marit Health. 2010; 61(1): 18-23, indexed in Pubmed: 20496323.

7. American College of Emergency Physicians (ACEP). Health Care Guidelines for Cruise Ship Medical Facilities. https://www.acep. org/Content.aspx?id=29980 (Revised July 2014).

8. Cruise Lines International Association (CLIA). Medical Facilities. https://www.cruising.org/about-the-industry/regulatory/ industry-policies/health/medical-facilities.

9. American Heart Association. Advanced cardiovascular life support - Provider manual. AHA, USA 2016 (ISBN 978-1-61669-400-5).

10. Sawyer T, Loren D, Halamek LP. Post-event debriefings during neonatal care: why are we not doing them, and how can we start? J Perinatol. 2016; 36(6): 415-419, doi: 10.1038/jp.2016.42, indexed in Pubmed: 27031321.

11. Devilly G, Gist R, Cotton P. Ready! Fire! Aim! The Status of Psychological Debriefing and Therapeutic Interventions: In the Work Place and After Disasters. Rev General Psychology. 2006; 10(4): 318-345, doi: 10.1037/1089-2680.10.4.318.

12. Mullan PC, Wuestner E, Kerr TD, et al. Implementation of an in situ qualitative debriefing tool for resuscitations. Resuscitation. 2013; 84(7): 946-951, doi: 10.1016/j.resuscitation.2012.12.005, indexed in Pubmed: 23266394.

13. Donabedian A. The quality of care - how can it be assessed? JAMA. 1988; 260(12): 1743-1748, doi: 10.1001/ jama.1988.03410120089033.

14. The Australian Transport Safety Bureau (ATSB) Interview protocols in transport safety investigations.https://www.atsb.gov.au/publications/2014/interview_protocols/.

15. Dahl E. Cruise ship's doctors - company employees or independent contractors? Int Marit Health. 2016; 67(3): 153-158, doi: 10.5603/ IMH.2016.0028, indexed in Pubmed: 27681214. 